\title{
ERRATA
}

\section{Author Correction}

The June 1, 2010, review article by Sideras et al, entitled "Coprescription of Tamoxifen and Medications That Inhibit CYP2D6” (J Clin Oncol 28:2768-2776, 2010), contained an error.

In Table 3, under the column "Moderate-to-Potent Inhibitors With Clearly Demonstrated or Expected In Vivo Inhibi- tion," the first medication for infectious diseases was given as "Terfenadine," whereas it should have been "Terbinafine."

The online version has been corrected in departure from the print. The authors apologize to the readers for the mistake.

DOI: $10.1200 /$ JCO.2010.31.0896

\section{Journal Corrections}

The March 1, 2010, article by Barlogie et al, entitled "LongTerm Follow-Up of Autotransplantation Trials for Multiple Myeloma: Update of Protocols Conducted by the Intergroupe Francophone du Myelome, Southwest Oncology Group, and University of Arkansas for Medical Sciences" (J Clin Oncol 28:1209-1214, 2010), contained errors.
In Figures 1-3, the first plot on the $\mathrm{x}$-axes was given as 10, whereas it should have been 4 , as follows: $0,4,8,12,16,20$.

Journal of Clinical Oncology apologizes to the authors and readers for the mistakes.

DOI: $10.1200 /$ JCO.2010.31.0862
The April 20, 2010, article by Perry et al, entitled "Phase II Trial of Continuous Dose-Intense Temozolomide in Recurrent Malignant Glioma: RESCUE Study" (J Clin Oncol 28:20512057, 2010), contained an error.

In the Results section, the last sentence of the third to last paragraph was given as: "Additionally, for each of the aforementioned subgroups, $23.1 \%, 24.2 \%, 7.7 \%$, and $25.9 \%$ and achieved stable disease for more than 6 months, for an overall clinical benefit of $38.5 \%, 27.3 \%, 7.7 \%$, and $7.0 \%$, respectively."
While it should have been: "Additionally, for each of the aforementioned subgroups, $23.1 \%, 24.2 \%, 7.7 \%$, and $25.9 \%$ and achieved stable disease for more than 6 months, for an overall clinical benefit of $38.5 \%, 27.3 \%, 7.7 \%$, and $37.0 \%$, respectively."

Journal of Clinical Oncology apologizes to the authors and readers for the mistake.

DOI: $10.1200 /$ JCO.2010.31.0870
The June 1, 2010, article by Hammond et al, entitled "American Society of Clinical Oncology/College of American Pathologists Guideline Recommendations for Immunohistochemical Testing of Estrogen and Progesterone Receptors in Breast Cancer" (J Clin Oncol 28:2784-2795, 2010), contained errors.

In the Recommendations section, under the heading "What Are the Clinically Validated Methods That Can Be Used in This Assessment?" and subheading "Laboratory concordance with standards," references 71 and 60 were cited in the sixth and seventh sentences of the first paragraph, whereas references 11 and 12 should have been cited, respectively.

In the same section, under the heading "What Are the Preanalytic, Analytic, and Postanalytic Variables That Must Be
Controlled to Ensure That the Assays Reflect the Tumor ER and PgR Status?" and subheading "Analytic standardization: antibody selection for ER testing," the antibody $1 \mathrm{~A} 6$ was inadvertently omitted from the third sentence, which should have read: "The Panel determined that the antibodies for ER that have met these criteria are clones 1D5, 6F11, SP1, and 1D5+ER.2.123, whereas the antibodies for PgR include clones 1A6, 1294, and 312 (Table 3)."

The online version has been corrected in departure from the print. Journal of Clinical Oncology and the authors apologize to the readers for the mistakes.

DOI: 10.1200/JCO.2010.31.0888 